\title{
Role of triaxiality in deformed halo nuclei
}

\section{$\operatorname{AUTHOR}(\mathrm{S})$ :}

Uzawa, K.; Hagino, K.; Yoshida, K.

\section{CITATION:}

Uzawa, K....[et al]. Role of triaxiality in deformed halo nuclei. Physical Review C 2021, 104(1): L011303.

ISSUE DATE:

2021-07

URL:

http://hdl.handle.net/2433/268038

RIGHT:

(C) 2021 American Physical Society 


\title{
Role of triaxiality in deformed halo nuclei
}

\author{
K. Uzawa, K. Hagino $\odot$, and K. Yoshida $\odot$ \\ Department of Physics, Kyoto University, Kyoto 606-8502, Japan
}

(Received 7 April 2021; revised 11 June 2021; accepted 20 July 2021; published 30 July 2021)

\begin{abstract}
It is known that nuclear deformation plays an important role in inducing the halo structure in neutron-rich nuclei by mixing several angular momentum components. While previous theoretical studies on this problem in the literature assume axially symmetric deformation, we here consider nonaxially symmetric deformations. With triaxial deformation, the $\Omega$ quantum number is admixed in a single-particle wave function, where $\Omega$ is the projection of the single-particle angular momentum on the symmetric axis, and the halo structure may arise even when it is absent with the axially symmetric deformation. In this way, the area of halo nuclei may be extended when triaxial deformation is considered. We demonstrate this idea using a deformed Woods-Saxon potential for nuclei with neutron number $N=13$ and 43 .
\end{abstract}

DOI: 10.1103/PhysRevC.104.L011303

With the developments of radioactive isotope beam facilities worldwide, many experimental data on neutron-rich nuclei have been accumulated. Among them, the halo phenomenon, first observed in ${ }^{11} \mathrm{Li}$ [1], has attracted lots of attention [2-8]. The halo nuclei are characterized by a spatially extended density distribution originated from weakly bound valence nucleon(s). Such structure can be probed, e.g., by interaction cross-section measurements [9] and the Coulomb dissociation reactions [7]. It has been shown that the valence nucleons have to have a low orbital angular momentum, either $l=0$ or $l=1$, in order for the halo structure to emerge [10-12]. With a higher angular momentum, the centrifugal barrier prevents the density distribution from extending, and the radius of a nucleus remains normal. As a consequence, $s$ and $p$ orbitals behave differently from orbitals with higher angular momentum, leading to a possible change in the shell structure [13-15].

Nuclear deformation significantly extends the region of halo nuclei [16-20]. When a nucleus is deformed, the orbital angular momentum is not a good quantum number anymore, and they are admixed in a single-particle wave function. With an axially symmetric deformation, single-particle states with $\Omega^{\pi}=\frac{1}{2}^{+}, \frac{1}{2}^{-}$, and $\frac{3}{2}^{-}$may then form a halo, where $\pi$ and $\Omega$ are parity and the projection of the total single-particle angular momentum $\boldsymbol{j}$ onto the symmetry axis, respectively, as these orbitals can contain the $s$-wave or the $p$-wave components. For instance, a $d_{3 / 2}$ orbital will couple to an $s_{1 / 2}$ orbital by nuclear deformation when $\Omega^{\pi}$ is equal to $\frac{1}{2}^{+}$, and a halo structure may arise even when the nucleus does not show it in the spherical limit. Moreover, it has been shown that the $s$-wave and $p$-wave components become dominant in a single-particle wave function when the separation energy is low $[16,17,21]$. The experimental indications of the halo structure in ${ }^{31} \mathrm{Ne}$ [22] and ${ }^{37} \mathrm{Mg}[23,24]$ are considered to be due to this mechanism, because the valence neutrons in these nuclei would occupy the $f_{7 / 2}$ orbital in the spherical limit [25-33].
We notice that all the discussions on deformed halo nuclei have so far been based on axially symmetric deformation. In this paper, we extend this to triaxial deformation, and clarify the role of triaxiality in deformed halo nuclei. When nonaxially symmetric deformation occurs, $\Omega$ is no longer a good quantum number and several components of $\Omega$ are mixed in a wave function. For instance, even though an orbital with $\Omega^{\pi}=\frac{5}{2}^{+}$does not form a halo structure with the axially symmetric deformation, the halo structure may arise in the presence of triaxial deformation, with mixing with an $\Omega^{\pi}=\frac{1}{2}^{+}$component. This is in a sense similar to an extension of the halo region going from spherical symmetry to axial deformation.

To demonstrate the role of triaxiality in deformed halo nuclei, let us consider a nucleus with neutron number $N=43$. For this purpose, we employ a triaxially deformed WoodsSaxon (WS) potential,

$$
V(\vec{r})=V_{0} f(\vec{r})-i V_{l s} r_{0}^{2}(\nabla f(\vec{r})) \cdot(\nabla \times \vec{s}),
$$

with

$$
f(\boldsymbol{r})=\frac{1}{1+\exp \{[r-R(\theta, \phi)] / a\}},
$$

where the radius parameter $R(\theta, \phi)$ is given by

$$
R(\theta, \phi)=R_{0}\left[1+\sum_{\mu} a_{2 \mu} Y_{2 \mu}^{*}(\theta, \phi)\right]
$$

Here, $a_{2 \mu}$ are the deformation parameters, $Y_{2 \mu}$ are the spherical harmonics, and $s$ is the spin operator. We use the parameters of $V_{0}=-42.86 \mathrm{MeV}, r_{0}=1.27 \mathrm{fm}, R_{0}=5.20$ $\mathrm{fm}, a=0.67 \mathrm{fm}$, and $V_{l s}=-0.44 V_{0}$. For simplicity, we here consider only the quadrupole deformation. In the following, we express the deformation using the parameters $\beta$ and $\gamma$, 


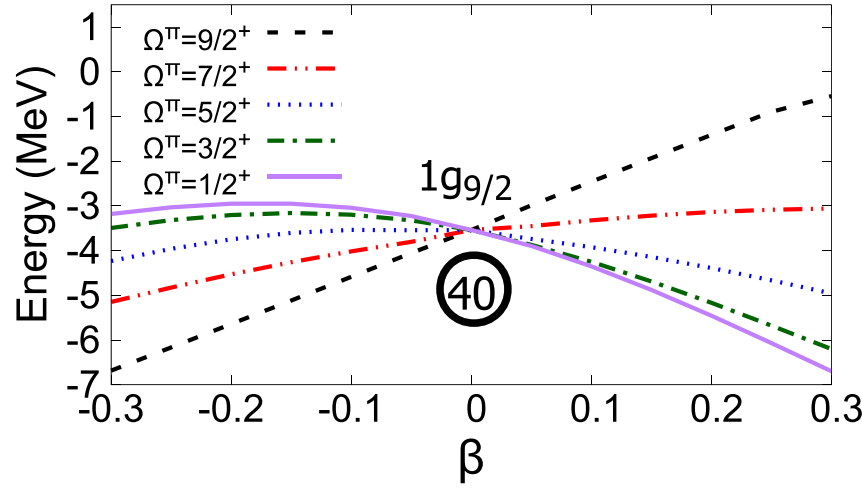

FIG. 1. Neutron levels as a function of the quadrupole deformation parameter $\beta$ obtained with an axially deformed Woods-Saxon potential.

instead of $a_{2 \mu}$, which are defined as

$$
\begin{aligned}
a_{20} & =\beta \cos \gamma, \\
a_{22} & =a_{2-2}=\frac{1}{\sqrt{2}} \beta \sin \gamma, \\
a_{2 \pm 1} & =0 .
\end{aligned}
$$

We solve the Schrödinger equation for single-particle wave functions with the three-dimensional (3D) mesh method [34]. We impose the parity symmetry and the $z$-signature symmetry $[34,35]$ so that we need to consider only the positive coordinate region: $x_{i}=(i-1 / 2) \Delta r, y_{j}=(j-1 / 2) \Delta r, z_{k}=$ $(k-1 / 2) \Delta r(i, j, k=1,2, \ldots)$ with the mesh size of $\Delta r=$ $0.9 \mathrm{fm}$ and 24 points for each direction. We have confirmed that results are almost converged with this box size, unless the $s$-wave component dominates in the single-particle wave functions.

Figure 1 shows the Nilsson diagram so obtained. In the spherical limit, the $43 \mathrm{rd}$ neutron occupies the $1 g_{9 / 2}$ orbit. With deformation, this level splits into five levels according to the value of $\Omega$. For a prolate deformation, the valence neutron occupies the orbital with $\Omega^{\pi}=\frac{3}{2}^{+}$, while the orbital with $\Omega^{\pi}=\frac{7}{2}^{+}$in the oblate region. Notice that neither of these does form a halo structure.

Let us then take into account the triaxiality $\gamma$. Figs. 2(a) and 2(b) show the fraction of the $s$-wave component $P_{s}$ and the expectation value of the $z$ component of the total single-particle angular momentum $\left\langle j_{z}\right\rangle$, respectively, for the single-particle level of the valence neutron. Different lines in the figures show the results of $\beta=0.3$ with several values of $\gamma$. To draw these figures, we vary $V_{0}$ in the Woods-Saxon potential to change the single-particle energy for the valence neutron. The fraction of the $s$-wave component is obtained by expanding the single-particle wave functions with the eigenfunctions of the spherical Woods-Saxon potential with $\beta=0$ in Eq. (1). We mention that $P_{s}$ does not reach $100 \%$ in the region shown in the figure, partly because we impose a boundary condition that the single-particle wave functions vanish at the edge of the box. For $\gamma=0^{\circ}$ and $\gamma=60^{\circ}, P_{s}$ is zero since the $\Omega^{\pi}=3 / 2^{+}$orbital has components with $j \geqslant 3 / 2$ and the $\Omega^{\pi}=7 / 2^{+}$orbital has components with $j \geqslant 7 / 2$

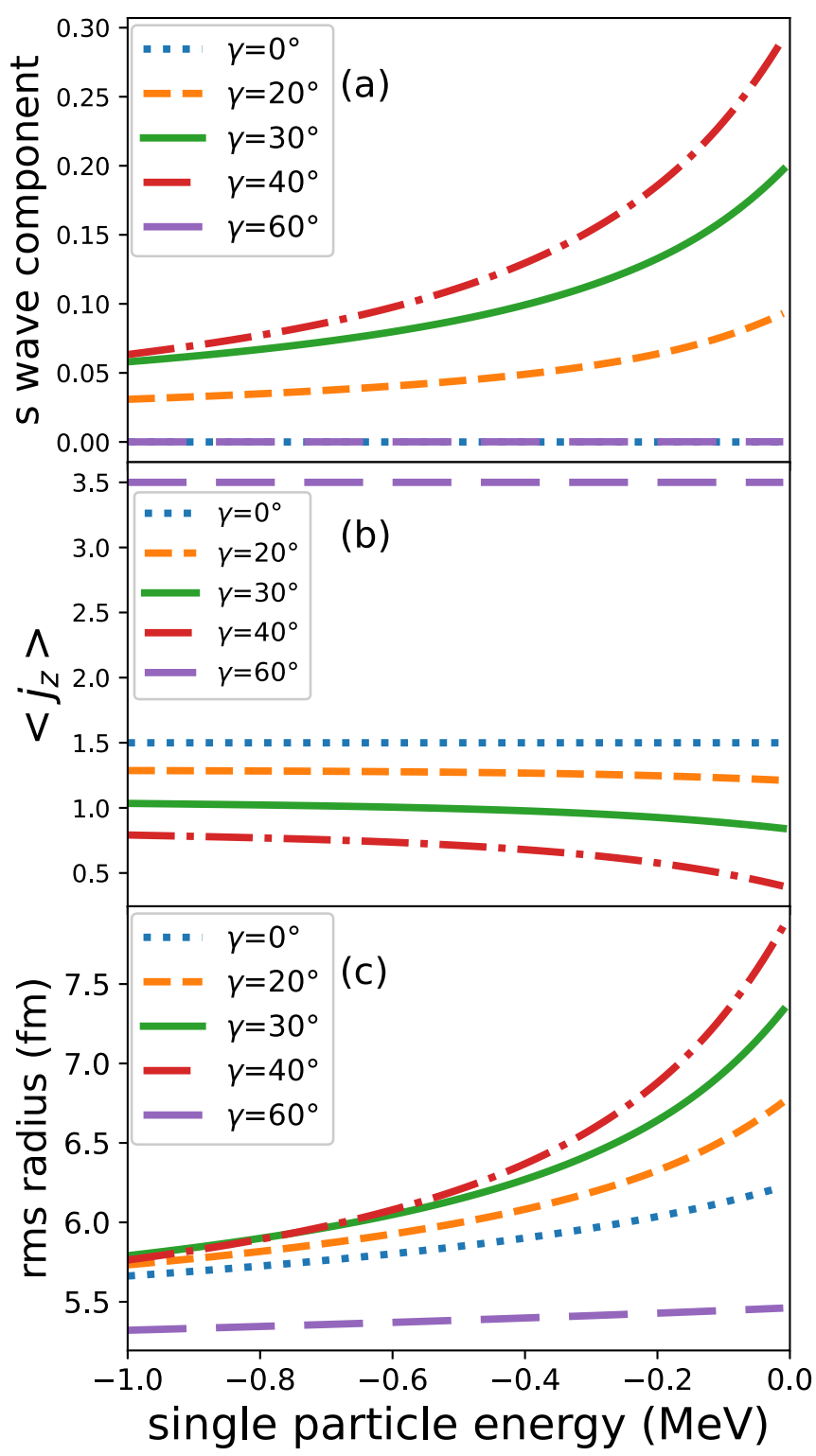

FIG. 2. (a) The fraction of the $s$-wave component, (b) the expectation value of the total single-particle angular momentum $j_{z}$, and (c) the root-mean-square (rms) radius for the 43rd neutron orbital in a deformed Woods-Saxon potential. These are plotted as a function of the single-particle energy for several values of triaxiality parameter $\gamma$ with $\beta=0.3$.

so $s_{1 / 2}$ cannot be mixed. As the triaxial deformation parameter $\gamma$ increases from $\gamma=0^{\circ}$ or decreases from $\gamma=60^{\circ}$, the $s$-wave component gradually increases. Accordingly, $\left\langle j_{z}\right\rangle$ decreases and deviates from the half-integer values, as is shown in Fig. 2(b). These features are also seen in Figs. 3(a) and 3(b), which show $P_{s}$ and $\left\langle j_{z}\right\rangle$ respectively, as a function of $\gamma$ at $S_{n}=0.2 \mathrm{MeV}$. One can see that with a triaxial deformation, the $s$-wave component increases significantly as the singleparticle energy approaches zero, and a nucleus may form a halo structure.

The root-mean-square (rms) radius $\left(\left\langle r^{2}\right\rangle\right)^{1 / 2}$ of the valence neutron is plotted in Fig. 2(c) as a function of the single-particle energy. As one expects from the 


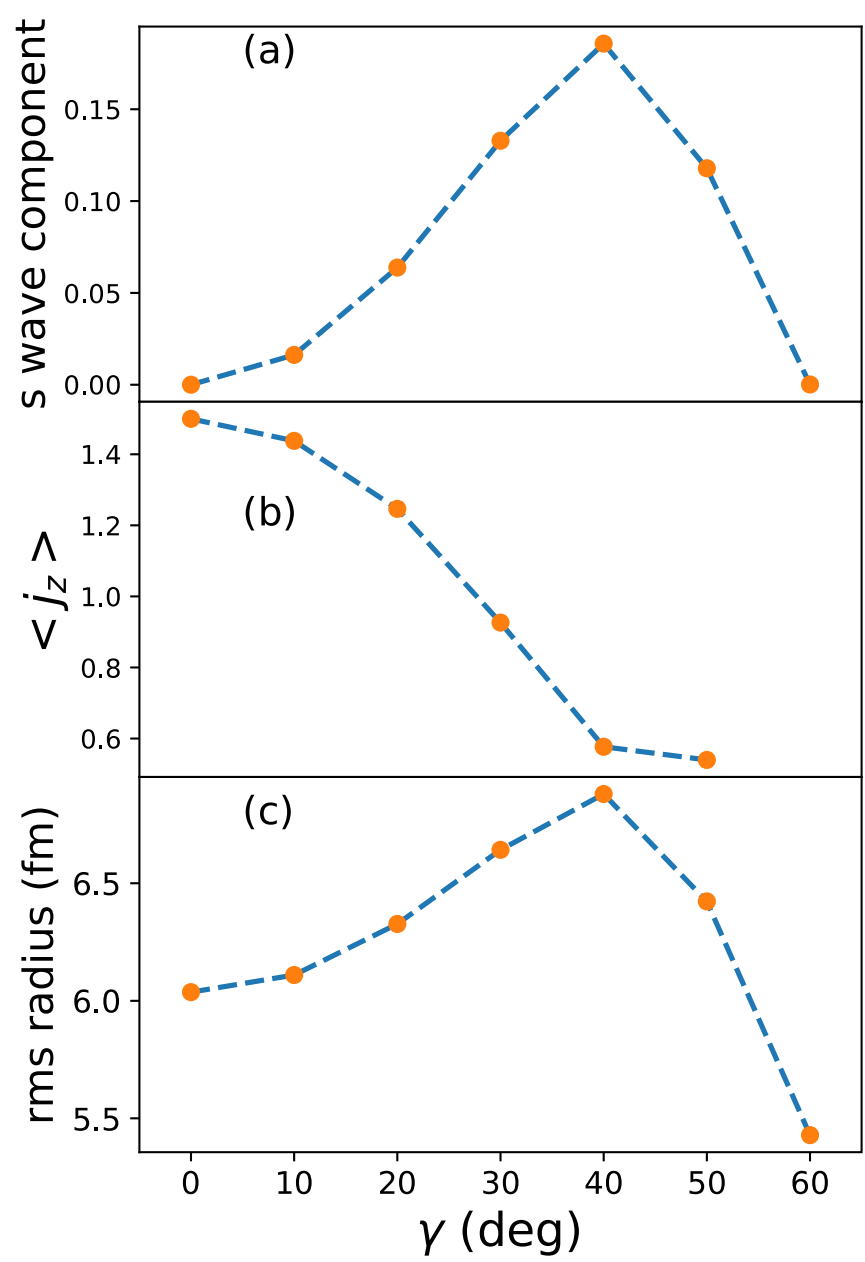

FIG. 3. Similar to Fig. 2, but as a function of $\gamma$ for $S_{n}=0.2 \mathrm{MeV}$.

behavior of the $s$-wave component, with the triaxial deformation the rms radius increases considerably as the single-particle energy approaches zero. Notice that this does not happen with axial deformation and triaxial deformation plays an essential role. In previous investigations of deformed halo nuclei, axial symmetry is assumed in most cases. Our work presented in this paper predicts that the region of halo nuclei can be extended if triaxial deformation emerges.

We next investigate briefly a nucleus with $N=13$, such as the ${ }^{19} \mathrm{C}$ nucleus, which is considered to be a one-neutron halo nucleus [9,36-43]. Figure 4 shows the Nilsson diagram for this nucleus. To this end, we use the parameters of $V_{0}=$ $-32.375 \mathrm{MeV}$ and $R_{0}=r_{0} A^{1 / 3}$ with $A=19$, while the other parameters are the same as those for Fig. 1 . For the ${ }^{19} \mathrm{C}$ nucleus, the valence neutron (i.e., the 13th neutron) occupies the orbital with $\Omega^{\pi}=\frac{1}{2}^{+}$and $\Omega^{\pi}=\frac{3}{2}^{+}$with a prolate and an oblate deformation, respectively. This orbital can thus form a halo in the prolate region while it cannot have the halo structure in the oblate region. With triaxial deformation, both of these aspects are mixed together.

Figure 5 shows the rms radius as a function of the singleparticle energy for $\beta=0.343$ with several values of $\gamma$. Here, $\beta=0.343$ is the optimized value for ${ }^{18} \mathrm{C}$ obtained with the

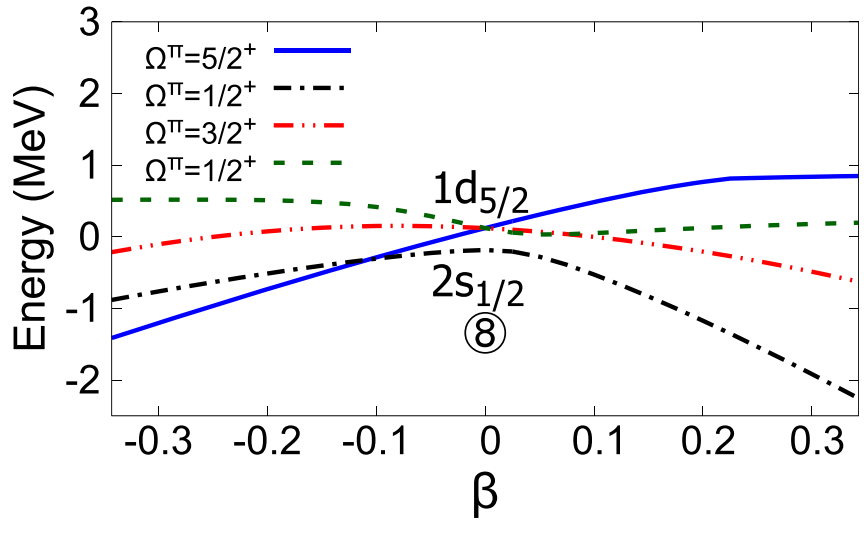

FIG. 4. A Nilsson diagram for neutron levels around $N=13$.

Skyrme-HF + BCS method [42]. In the figure, the boxes with right diagonal lines and left diagonal lines denote the experimental radius $\left(\left\langle r^{2}\right\rangle\right)^{1 / 2}=5.5 \pm 0.3 \mathrm{fm}[36]$ and $\left(\left\langle r^{2}\right\rangle\right)^{1 / 2}=$ $6.4 \pm 0.7 \mathrm{fm}$ [38], respectively, together with the one-neutron separation energy $S_{n}=160 \pm 110 \mathrm{keV}$ obtained with the direct mass measurements [44]. The figure implies that the calculation with $\gamma=60^{\circ}$ underestimates the rms radius, while the calculation with $\gamma=0^{\circ}$ clearly overestimates the rms radius. By taking into account the triaxiality in the deformation, the halo component is modified in the wave function and the rms radius and the one-neutron separation energy are simultaneously reproduced with $\gamma=20^{\circ}-50^{\circ}$.

In summary, we have introduced a new perspective of deformed halo nuclei by considering triaxial deformation; we have pointed out that the mixing of configurations with different $\Omega$ quantum numbers with nonaxially symmetric deformations may lead to the halo structure even when it does not appear with axially symmetric deformation. We have demonstrated this using neutron orbitals for the 43rd and the 13th neutrons in a deformed Woods-Saxon

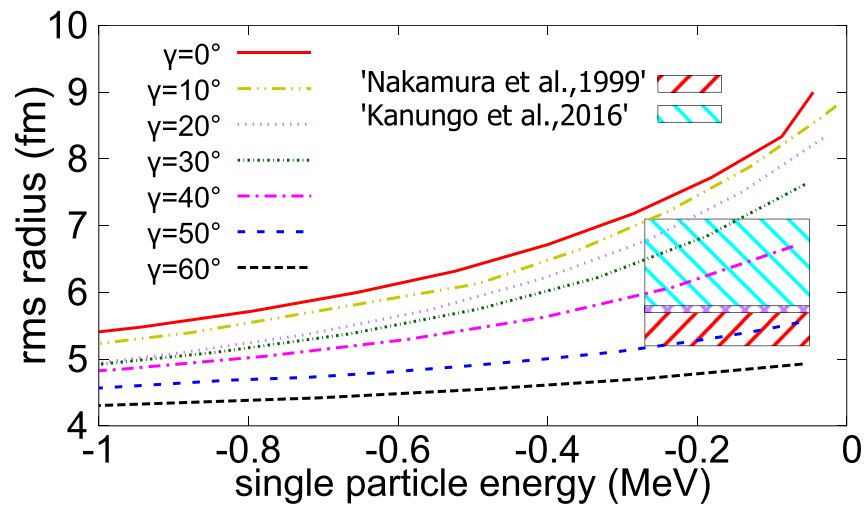

FIG. 5. Relation between the single-particle energy of the valence neutron in ${ }^{19} \mathrm{C}$ and the root-mean-square (rms) radius for several values of the triaxial deformation parameter $\gamma$. The deformation parameter $\beta$ is fixed to be 0.343 . The boxes with the right diagonal lines and the left diagonal lines show the experimental values of the rms radius from Nakamura et al. (1999) [36] and Kanungo et al.(2016) [38], respectively, together with the empirical separation energy $S_{n}=160 \pm 110 \mathrm{keV}$ from Ref. [44]. 
potential. The finding in this paper would enlarge the region of halo nuclei if triaxial deformation appears in neutron-rich medium-heavy and heavy nuclei. It would be an interesting future work to carry out a systematic study of deformed halo nuclei in a wide range of the nuclear chart by taking into account triaxial deformations. In this connection, we mention that recent finite range droplet model (FRDM) calculations have indicated that medium-heavy nuclei near the drip lines deform triaxially in the wide region of the periodic table [45]. In addition, symmetry unrestricted HF +
BCS calculations have shown that nuclei near the drip lines with $N=60-80$ and $N=110-120$ tend to be triaxially deformed [46].

We thank Nigel Orr for useful discussions on the direct mass measurements of ${ }^{19} \mathrm{C}$. This work was supported by JSPS KAKENHI (Grants No. JP19K03824, No. JP19K03861, and No. JP19K03872). The numerical calculations were performed with the computing facility at the Yukawa Institute for Theoretical Physics, Kyoto University.
[1] I. Tanihata, H. Hamagaki, O. Hashimoto, Y. Shida, N. Yoshikawa, K. Sugimoto, O. Yamakawa, T. Kobayashi, and N. Takahashi, Phys. Rev. Lett. 55, 2676 (1985).

[2] M. V. Zhukov, B. V. Danilin, D. V. Fedorov, J. M. Bang, I. J. Thompson, and J. S. Vaagen, Phys. Rep. 231, 151 (1993).

[3] A. S. Jensen, K. Riisager, D. V. Fedorov, and E. Garrido, Rev. Mod. Phys. 76, 215 (2004).

[4] B. Jonson, Phys. Rep. 389, 1 (2004).

[5] K. Hagino, I. Tanihata, and H. Sagawa, in 100 Years of Subatomic Physics, edited by E. M. Henley and S. D. Ellis (World Scientific, Singapore, 2013), p. 231.

[6] I. Tanihata, H. Savajols, and R. Kanungo, Prog. Part. Nucl. Phys. 68, 215 (2013).

[7] T. Nakamura, H. Sakurai, and H. Watanabe, Prog. Part. Nucl. Phys. 97, 53 (2017).

[8] T. Otsuka, A. Gade, O. Sorlin, T. Suzuki, and Y. Utsuno, Rev. Mod. Phys. 92, 015002 (2020).

[9] A. Ozawa, T. Suzuki, and I. Tanihata, Nucl. Phys. A 693, 32 (2001).

[10] K. Riisager, A. S. Jensen, and P. Møller, Nucl. Phys. A 548, 393 (1992).

[11] H. Sagawa, Phys. Lett. B 286, 7 (1992).

[12] H. Sagawa and K. Hagino, Eur. Phys. J. A 51, 102 (2015).

[13] I. Hamamoto, S. V. Lukyanov, and X. Z. Zhang, Nucl. Phys. A 683, 255 (2001).

[14] I. Hamamoto, J. Phys. G 37, 055102 (2010).

[15] I. Hamamoto, Phys. Rev. C 85, 064329 (2012).

[16] T. Misu, W. Nazarewicz, and S. Åberg, Nucl. Phys. A 614, 44 (1996).

[17] I. Hamamoto, Phys. Rev. C 69, 041306(R) (2004).

[18] S. G. Zhou, J. Meng, P. Ring, and E. G. Zhao, Phys. Rev. C 82, 011301(R) (2010).

[19] I. Hamamoto, Phys. Rev. C 95, 044325 (2017).

[20] I. Hamamoto, Phys. Lett. B 814, 136116 (2021).

[21] K. Yoshida and K. Hagino, Phys. Rev. C 72, 064311 (2005).

[22] T. Nakamura et al., Phys. Rev. Lett. 103, 262501 (2009).

[23] M. Takechi et al., Phys. Rev. C 90, 061305(R) (2014).

[24] N. Kobayashi et al., Phys. Rev. Lett. 112, 242501 (2014).
[25] I. Hamamoto, Phys. Rev. C 81, 021304(R) (2010).

[26] K. Minomo, T. Sumi, M. Kimura, K. Ogata, Y. R. Shimizu, and M. Yahiro, Phys. Rev. C 84, 034602 (2011).

[27] K. Minomo, T. Sumi, M. Kimura, K. Ogata, Y. R. Shimizu, and M. Yahiro, Phys. Rev. Lett. 108, 052503 (2012).

[28] T. Sumi, K. Minomo, S. Tagami, M. Kimura, T. Matsumoto, K. Ogata, Y. R. Shimizu, and M. Yahiro, Phys. Rev. C 85, 064613 (2012).

[29] Y. Urata, K. Hagino, and H. Sagawa, Phys. Rev. C 86, 044613 (2012)

[30] S. Watanabe et al., Phys. Rev. C 89, 044610 (2014).

[31] Y. Urata, K. Hagino, and H. Sagawa, Phys. Rev. C 96, 064311 (2017).

[32] H. Nakada and K. Takayama, Phys. Rev. C 98, 011301(R) (2018).

[33] H. Kasuya and K. Yoshida, Prog. Theor. Exp. Phys. 2021, 013D01 (2021).

[34] P. Bonche, H. Flocard, P. H. Heenen, S. J. Krieger, and M. S. Weiss, Nucl. Phys. A 443, 39 (1985).

[35] H. Ogasawara, K. Yoshida, M. Yamagami, S. Mizutori, and K. Matsuyanagi, Prog. Theor. Phys. 121, 357 (2009).

[36] T. Nakamura et al., Phys. Rev. Lett. 83, 1112 (1999).

[37] D. Bazin et al., Phys. Rev. Lett. 74, 3569 (1995).

[38] R. Kanungo et al., Phys. Rev. Lett. 117, 102501 (2016).

[39] J. S. Wang et al., Nucl. Phys. A 691, 618 (2001).

[40] X.-N. Cao, Q. Liu, and J.-Y. Guo, J. Phys. G 45, 085105 (2018).

[41] X.-X. Sun, J. Zhao, and S.-G. Zhou, Nucl. Phys. A 1003, 122011 (2020).

[42] Y. Zhang, H. Sagawa, D. Yoshino, K. Hagino, and J. Meng, Prog. Theor. Phys. 120, 129 (2008).

[43] Y. Kanada-En'yo, Phys. Rev. C 71, 014310 (2005).

[44] G. Audi and A. H. Wapstra, Nucl. Phys. A 565, 1 (1993).

[45] P. Möller, R. Bengtsson, B. G. Carlsson, P. Olivius, T. Ichikawa, H. Sagawa, and A. Iwamoto, At. Data Nucl. Data Tables 94, 758 (2008).

[46] S. Ebata and T. Nakatsukasa, Phys. Scr. 92, 064005 (2017); https://wwwnucl.ph.tsukuba.ac.jp/InPACS. 